\title{
TENSOR PRODUCTS OF SIMPLE PURE INSEPARABLE FIELD EXTENSIONS
}

\author{
J. N. MORDESON AND B. VINOGRADE ${ }^{1}$
}

Let $K$ be a field of characteristic $p \neq 0$ and let $L$ be a pure inseparable extension field of finite degree over $K$. Our purpose is to give several necessary and sufficient conditions for $L$ to be a tensor product of simple extensions over $K$. Weisfeld [4] has a criterion, namely the existence of a nontrivial higher derivation of $L$ with $K$ as its subfield of constants, (in fact Weisfeld proves his criterion for infinite extensions of bounded exponent). The present note describes different criteria, in terms of Pickert's canonical generators [3, p. 133]. For a given canonical generating set $\left\{b_{1}, \cdots, b_{r}\right\}$ of $L$ over $K$, let $M_{i}$ $=K\left(b_{1}, \cdots, b_{i}\right)$ and let $q_{i}$ denote $p^{e_{i}}$ where $e_{i}$ is the exponent of $b_{i}$ over $M_{i-1}, i=1, \cdots, r$, where $M_{0}=K$. We shall prove the following theorem.

THEOREM. If $L$ is a finite degree pure inseparable extension of $K$, then the following conditions are equivalent:

(0) $L$ is the tensor product of a finite number of simple extensions with respect to $K$.

(1) Every canonical generating set is such that

$$
b_{i}^{q_{i}} \in\left(L^{q_{i}} \cap K\right)\left(b_{1}^{q_{i}}, \cdots, b_{i-1}^{q_{i}}\right)=M_{i-1}^{q_{i}}\left(L^{q_{i}} \cap K\right), \quad i=1, \cdots, r .
$$

(2) Every canonical generating set is such that the tensor product $L \otimes M_{i}$ with respect to $K$ cleaves over $1 \otimes M_{i}$ (that is, $L \otimes M_{i}$ has a Wedderburn factor as an algebra over $\left.1 \otimes M_{i}\right), i=1, \cdots, r$.

(3) There exists a canonical generating set such that $L \otimes M_{i}$ cleaves over $1 \otimes M_{i}, i=1, \cdots, r$.

(4) There exists a canonical generating set such that $b_{i}^{q_{i}} \in M_{i-1}^{q_{i}}\left(L^{q_{i}} \cap K\right)$, $i=1, \cdots, r$.

Proof. (0) implies (1): Suppose $L \cong K\left(a_{1}\right) \otimes \cdots \otimes K\left(a_{r}\right)$ and that $\left\{a_{1}, \cdots, a_{r}\right\}$ is already ordered so that it is a canonical generating set. Let $\left\{b_{1}, \cdots, b_{r}\right\}$ be any given canonical generating set. For any $c \in L, \quad c^{q_{i}}=\left(\sum_{j} k_{j} a_{1}^{j_{1}} \cdots a_{r}^{j_{r}}\right)_{q_{i}}$ where $k_{j} \in K$ and $j=\left\{j_{1}, \cdots, j_{r}\right\}$. By the division algorithm, $a_{n}{ }^{j_{n} q_{i}}=a_{n}{ }_{n} q_{n} a_{n}{ }^{r_{n}}$ where $0 \leqq r_{n}<q_{n}(n=1$, $\cdots, i-1)$. Since $q_{i}$ divides $q_{n}, r_{n}$ has the form $q_{i} t_{n}(n=1, \cdots, i-1)$. Thus, since $\left\{a_{i}^{q_{i}}, \cdots, a_{r}^{q_{i}}\right\} \subseteq K$ and $\left\{a_{1}^{q_{1}}, \cdots, a_{i-1}^{q_{i-1}}\right\} \subseteq L^{q_{i}} \cap K$, there

Received by the editors September 8, 1967.

1 This paper was partially supported by NSF Grant G-23418. 
exists a set $\left\{k_{t}^{\prime}\right\} \subseteq L^{q_{i}} \cap K$ such that for $t=\left\{t_{1}, \cdots, t_{i-1}\right\}$,

(*) $\quad c^{q_{i}}=\sum_{t} k_{t}^{\prime} a_{1}^{q_{i} t_{1}} \cdots a_{i-1}^{q_{i} t_{i-1}}, \quad 0 \leqq q_{i} t_{n}<q_{n}(n=1, \cdots, i-1)$.

Since the monomials $\left\{a_{1}^{q_{i} t_{1}} \cdots a_{i-1}^{q_{i} t_{i-1}}\right\}$ are linearly independent over $K$, this set $\left\{k_{j}^{\prime}\right\}$ is the only subset of $K$ satisfying (*). In particular,

$$
b_{i}^{q_{i}}=\sum_{t} k_{t} a_{1}^{q_{i} t_{1}} \cdots a_{i-1}^{q_{i} t_{i-1}}, \quad k_{t} \in L^{q_{i}} \cap K, \quad t=\left\{t_{1}, \cdots, t_{i-1}\right\}
$$

and $0 \leqq q_{i} t_{n}<q_{n}(n=1, \cdots, i-1)$. Also,

$$
b_{i}^{q_{i}}=\sum_{s} k_{s}^{\prime \prime} b_{1}^{q_{i} s_{1}} \cdots b_{i-1}^{q_{i} s_{i-1}}, \quad k_{s}^{\prime \prime} \in K, \quad s=\left\{s_{1}, \cdots, s_{i-1}\right\}
$$

and

$$
0 \leqq q_{i} s_{n}<q_{n} \quad(n=1, \cdots, i-1) .
$$

Thus, by (*),

$$
\begin{aligned}
b_{i}^{q_{i}} & =\sum_{s} k_{s}^{\prime \prime}\left(b_{1}^{s_{1}} \cdots b_{i-1}^{s_{i-1}}\right)^{q_{i}} \\
& =\sum_{s} k_{s}^{\prime \prime}\left(\sum_{t} k_{s t} a_{1}^{q_{i} t_{1}} \cdots a_{i-1}^{q_{i} t_{i-1}}\right),
\end{aligned}
$$

$k_{s t} \in L^{q_{i}} \cap K$ and $0 \leqq q_{i} t_{n}<q_{n}(n=1, \cdots, i-1)$. Therefore, by $(* *)$ and $(* * *), k_{t}=\sum_{s} k_{s}^{\prime \prime} k_{s t}$ for each $t$. Since the set $\left\{k_{s}^{\prime \prime}\right\}$ exists, the system $k_{t}=\sum_{s} x_{s} k_{s t}$ has a solution in $L^{q_{i}} \cap K$, say $x_{s}=k_{s}^{*} \in L^{q_{i}} \cap K$. Hence,

$$
\begin{aligned}
b_{i}^{q_{i}} & =\sum_{t}\left(\sum_{s} k_{s}^{*} k_{s t}\right) a_{1}^{q_{i} t_{1}} \cdots a_{i-1}^{q_{i} t_{s-1}} \\
& =\sum_{s} k_{s}^{*}\left(\sum_{t} k_{s t} a_{1}^{q_{i} t_{1}} \cdots a_{i-1}^{q_{i} t_{s}-1}\right) \\
& =\sum_{t} k_{s}^{*}\left(b_{1}^{s_{1}} \cdots b_{i-1}^{s_{i-1}}\right)^{q_{i}} \in M_{i-1}^{q_{i}}\left(L^{q_{i}} \cap K\right) .
\end{aligned}
$$

(4) implies (0): Make the induction hypothesis that $L \cong M_{i} \otimes M_{i}^{\prime}$ where $M_{i}^{\prime}=K\left(a_{i+1}\right) \otimes \cdots \otimes K\left(a_{r}\right)$ (there being nothing to prove for $i=r)$. Since $b_{i}^{q_{i}}=\sum_{j} k_{j} b_{1}^{q_{i} j_{1}} \cdots b_{i-1}^{q_{i} j_{i-1}}$ where $k_{j}=c_{j}^{q_{i}} \in L^{q_{i}} \cap K$ and $j=\left\{j_{1}, \cdots, j_{i-1}\right\}$, we have $b_{i}=\sum_{j} c_{j} b_{1}^{j_{1}} \cdots b_{i-1}^{j_{i-1}}$. Hence, $M_{i}$ $=M_{i-1}\left(b_{i}\right)=M_{i-1}\left(\left\{c_{j}\right\}\right)$. Since $M_{i}$ is simple pure inseparable over $M_{i-1}$, there exists $a_{i} \in\left\{c_{j}\right\}$ such that $M_{i}=M_{i-1}\left(a_{i}\right)$ and $a_{i}^{q_{i}} \in K$. Since $\left[M_{i}: K\right]=q_{1} \cdots q_{i}$, it follows that $\left[M_{i-1}\left(a_{i}\right): M_{i-1}\right]=q_{i}$. 
Hence, $\quad M_{i} \cong M_{i-1} \otimes K\left(a_{i}\right)$. Thus, $L \cong M_{i-1} \otimes M_{i-1}^{\prime}$ where $M_{i-1}^{\prime}$ $=K\left(a_{i}\right) \otimes \cdots \otimes K\left(a_{r}\right)$. Hence, by induction, $L \cong K\left(a_{1}\right) \otimes \cdots$ $\otimes K\left(a_{r}\right)$. (E. A. Hamann has a different proof of this implication.)

Since (1) implies (4) trivially, we have the equivalence of (0), (1) and (4).

(1) implies (2): Since $b_{i}^{q} \in M_{i-1}^{q_{i}}\left(L^{q_{i}} \cap K\right), i=1, \cdots, r$, we have $b_{i}=\sum_{j} c_{j} m_{j}$ where $c_{j} \in L, \quad m_{j} \in M_{i-1}$ and $c_{j}^{q_{i}}=k_{j} \in K$. Let $b_{i}^{\prime}$ $=\sum_{j} c_{j} \otimes m_{j}$. Then $b_{i}^{\prime q_{i}}=\sum_{j} c_{j}^{q} \otimes m_{j}^{q}=\sum_{j} 1 \otimes k_{j} m_{j}^{q_{i}} \in 1 \otimes M_{i-1}$. Since $e_{i}$ is the exponent of $b_{i}$ over $M_{i-1}, M_{i}^{\prime}=\left(1 \otimes M_{i-1}\right)\left[b_{i}^{\prime}\right]$ is a field $(i=1, \cdots, r)$.

Now consider $L \otimes M_{i}$ for any $i=1, \cdots, r$. Suppose there exists a field $M_{j}^{*}$ in $L \otimes M_{i}$ such that $M_{j}^{*} \supseteq 1 \otimes M_{i}(j \geqq i)$ and $f_{i} M_{j}^{*}=M_{j}$ where $f_{i}$ is the canonical $K$-epimorphism of $L \otimes M_{i}$ onto $L M_{i}=L$. By the previous paragraph, there exists a field $M_{j+1}^{\prime} \subseteq L \otimes M_{j}$ such that $M_{j+1}^{\prime} \supseteq 1 \otimes M_{j}$ and $f_{j} M_{j+1}^{\prime}=M_{j+1} \subseteq L$. By the universal mapping theorem for tensor products, there exists a $K$-epimorphism $h_{j}$ of $L \otimes M_{j}$ onto the ring composite $\left[L \otimes 1, M_{j}^{*}\right] \subseteq L \otimes M_{i}$ such that $f_{j}=f_{i} h_{j}$. Thus, there exists a field $M_{j+1}^{*}$ in $L \otimes M_{i}$ such that $M_{j+1}^{*} \supseteq M_{j}^{*} \supseteq 1 \otimes M_{i}$ and $f_{i} M_{j+1}^{*}=M_{j+1} \subseteq L$, namely the field $M_{j+1}^{*}=h_{j} M_{j+1}^{\prime}$. Hence, the proof follows by induction.

(2) implies (3): Immediate.

(3) implies (4): Let $\left\{b_{1}, \cdots, b_{r}\right\}$ be any canonical generating set such that $L \otimes M_{i}$ cleaves over $1 \otimes M_{i}, i=1, \cdots, r$. Use the symbol $\otimes_{1}$ to denote the tensor product with respect to $M_{1}$. Then there is a canonical $K$-epimorphism of $L \otimes M_{i}$ onto $L \otimes_{1} M_{i}$, whence $L \otimes_{1} M_{i}$ cleaves over $1 \otimes_{1} M_{i}, i=1, \cdots, r$. Now make the induction hypothesis that (3) implies (4) for all pure inseparable extensions of multiplicity less than $r$. ((3) implies (4) trivially for $r=1$.) Then since we have proved (4) is equivalent to $(0), L \cong M_{1}\left(b_{2}^{\prime}\right) \otimes_{1} \cdots \otimes_{1} M_{1}\left(b_{r}^{\prime}{ }^{\prime}\right)$ and we may assume $\left\{b_{2}^{\prime}, \cdots, b_{r}^{\prime}\right\}$ is canonically ordered over $M_{1}$. Since $b_{1}$ has maximal exponent in $L$ over $K$ and $b_{i}^{\prime}$ has maximal exponent in $L$ over $M_{i-1}^{\prime}\left(M_{1}^{\prime}=M_{1}=K\left(b_{1}\right)\right.$ and $M_{j}^{\prime}=M_{1}\left(b_{2}^{\prime}, \cdots, b_{j}^{\prime}\right)$, $\left.j=2, \cdots, r^{\prime}\right)$, it follows that $\left\{b_{1}, b_{2}^{\prime}, \cdots, b_{r}^{\prime}\right\}$ is a canonical generating set of $L$ over $K$, whence $r=r^{\prime}$. In particular,

$$
b_{j}^{\prime q_{j}} \in K\left(b_{1}^{q_{j}}, b_{2}^{\prime q_{j}}, \cdots, b_{j-1}^{\prime q_{j}}\right) \cap M_{1}, \quad j=2, \cdots, r
$$

since the $e_{i}$ of a canonical generating set are invariant. Because $L \otimes M_{1}$ cleaves over $1 \otimes M_{1}$, there exists $b_{j}^{*} \in L \otimes M_{1}$ such that $f_{1} b_{j}^{*}=b_{j}^{\prime}$ and $b_{j}^{*} a_{j} \in 1 \otimes M_{1}, j=2, \cdots, r$. Now $b_{j}^{*}=\sum_{8} c_{8} \otimes b_{1}^{s}, c_{s} \in L$, whence $b_{j}^{*} a_{j}=\sum_{s} c_{s} q_{i} \otimes b_{1} q_{j^{s}}$. By the division algorithm, $b_{1} q_{j}^{s}=b_{1} q_{1} n_{s} b_{1} r_{c}$ where $0 \leqq r_{s}<q_{1}$. Since $b_{1}^{q_{1} n_{s}} \in K$ and $q_{j}$ divides $q_{1}$, it follows that 
$b_{j}^{*} q_{j}=\sum_{s} c_{s}^{\prime} q_{j} \otimes b_{1^{s}}^{r_{s}}$ where $c_{s}^{\prime} \in L$. Also, $b_{j}^{*_{q_{j}}}=\sum_{s} 1 \otimes k_{s} b_{1}^{q^{s_{1}} b_{2} q_{j} s_{2}} \ldots$ $b_{j-1}^{\prime \prime g s_{j-1}}, s=\left\{s_{1}, \cdots, s_{j-1}\right\}$, since $M_{1} \subseteq M_{j}^{\prime}$. Therefore, $k_{s} \in L^{q_{j}} \cap K$ and $b_{j}^{\prime q_{j}} \in M_{j-1}^{\prime q}\left(L^{q_{j}} \cap K\right)$. Hence, $\left\{b_{1}, b_{2}^{\prime}, \cdots, b_{r}^{\prime}\right\}$ is a canonical generating set satisfying (4). Q.E.D.

Examples where $L$ is not a tensor product of simple extensions can be found in [1, Ex. 6, p. 196] and [2, p. 51]. If $\left\{b_{1}, \cdots, b_{r}\right\}$ is a canonical generating set satisfying (4), it does not follow that $L$ $\cong K\left(b_{1}\right) \otimes \cdots \otimes K\left(b_{r}\right)$. For example, consider a perfect field $P$ and independent indeterminates $s, t$ over $P$. Let $K=P(s, t)\left(s^{1 / p}+t^{1 / p}\right)$ and $L=P\left(s^{1 / p^{2}}, t^{1 / p^{2}}\right)$. If $b_{1}=s^{1 / p^{2}}$ and $b_{2}=t^{1 / p^{2}}$, then $\left\{b_{1}, b_{2}\right\}$ is a canonical generating set with $e_{1}=2$ and $e_{2}=1$. It is easily verified that $b_{2}^{Q_{2}}$ $\in\left(L^{q^{2}} \cap K\right)\left(b^{q^{2}}\right)$, but $L \not \equiv K\left(b_{1}\right) \otimes K\left(b_{2}\right)$ since $b_{2}$ has exponent 2 over $K$. However, $L \cong K\left(s^{1 / p^{2}}\right) \otimes K\left(s^{1 / p^{2}}+t^{1 / p^{2}}\right)$.

The extent to which these results are valid in arbitrary pure inseparable extensions is considered by the authors in an article to appear in the Mathematische Zeitschrift. Other recent results can be found in an article by Haddix and Mordeson in the Formosan Science and in an article by Sweedler in the Annals. The equivalence of $(0)$ above and the linear disjointness of $K$ and $L^{p^{i}}(i=1,2, \cdots)$ is proved independently in these articles.

\section{REFERENCES}

1. N. Jacobson, "Theory of fields and Galois theory" in Lectures in abstract algebra, Vol. 3, Van Nostrand, Princeton, N. J., 1964.

2. J. N. Mordeson and B. Vinograde, Extension of certain subfields to coefficient fields in commutative algebras, J. Math. Soc. Japan 17 (1965), 47-51.

3. G. Pickert, Eine Normalform für endliche rein-inseparable Körpererweiterungen, Math. Z. 53 (1950), 133-135.

4. M. Weisfeld, Purely inseparable extensions and higher derivations, Trans. Amer. Math. Soc. 116 (1965), 435-449.

Creighton University and Iowa State University 